\title{
A Proposal to Operate the COUPP-60 Bubble Chamber at SNOLAB
}

\author{
Juan Collar [spokesman] (PI), C. Eric Dahl, Drew Fustin, Luke Goetzke, \\ Nathan Riley, Hannes Schimmelpfennig, Matthew Szydagis \\ Kavli Institue for Cosmological Physics \\ University of Chicago
}

Ed Behnke, Henry Hinnefeld, Ilan Levine(PI), Andrea Palenchar, Tina Marie Shepherd, Brendan Sweeney

Department of Physics

Indiana University South Bend

Stephen Brice, Daniel Broemmelsiek, Peter Cooper, Michael Crisler, Jeter Hall, Erik Ramberg, Andrew Sonnenschein, Robert Tschirhart

Fermi National Accelerator Laboratory

August 242009

\begin{abstract}
Bubble chambers are promising devices for the detection of WIMP dark matter, due to their easy scalability to large target masses and insensitivity to background $\gamma$ and $\beta$ radiation. The COUPP collaboration has constructed small chambers which have achieved competitive sensitivity for spin-dependent WIMP-nucleon scattering. A new chamber, COUPP-60, containing $60-\mathrm{kg}$ of $\mathrm{CF}_{3} \mathrm{I}$ target liquid, has been built and is being commissioned at Fermilab. We propose to move this detector to SNOLAB after completing tests in a shallow underground site at Fermilab. At SNOLAB, we expect the sensitivity of the experiment to be determined by the level of $\alpha$-emitting contamination in the target liquid. If we achieve state-of-the-art levels of $\alpha$ emitting contamination, we will improve current sensitivity by approximately four orders of magnitude beyond our published limits, to the region of $10^{-4} \mathrm{pb}$ for a $30 \mathrm{GeV}$ WIMP interacting by spindependent couplings to the proton. This will allow a first exploration of the phase space favored by supersymmetric models in this regime.
\end{abstract}




\section{Introduction}

COUPP-60, Fermilab E961, is an experiment with the goal of detecting dark matter in the form of Weakly Interacting Massive Particles (WIMPs) by observing the single bubbles produced by recoils of nuclei struck by dark matter particles. [1] The detector is a continuously sensitive bubble chamber, operated at mildly superheated conditions, which is sensitive only to the highly concentrated energy deposition from nuclear recoils. The fluid used is the fluorocarbon $\mathrm{CF}_{3} \mathrm{I}$. The operating temperature is near 40 degrees $C$, with operating pressures from about 5 psig up to about 90 psig. This range corresponds to an energy threshold for the detection of nuclear recoils from $5 \mathrm{keV}$ to several hundred $\mathrm{keV}$. At the lowest threshold this detector will be sensitive to most nuclear recoils from WIMPs with masses greater than $10 \mathrm{GeV}$. At the highest pressure, the threshold is above the range expected for nuclear recoils from WIMPs. The integral spectrum as a function of threshold energy has a specific shape for a given WIMP mass, which can be used to discriminate WIMPs from backgrounds.

The COUPP-60 detector was built in the period 2007-2009 and is now being commissioned. We expect the commissioning phase at Fermilab to continue through mid-2010. To achieve its full potential sensitivity, this detector must be operated at a deep underground site, where backgrounds from cosmic-ray induced neutrons will become negligible. Therefore, we propose to move the detector to SNOLAB once commissioning milestones have been achieved.

At SNOLAB, we expect the sensitivity of the experiment to be determined by the level of $\alpha$-emitting contamination in the target liquid. If we achieve state-of-the-art levels of purity, we will improve current sensitivity substantially more than three orders of magnitude, to the region of a $10^{-4} \mathrm{pb}$ cross section for a $30 \mathrm{GeV}$ WIMP interacting by spin-dependent couplings to the proton. This will allow a first exploration of the phase space favored by supersymmetric models for this coupling. [2] The supersymmetric parameters that can be probed are not the same as those presently eliminated by XENON and CDMS for spin-independent couplings.[3]

\section{Design of the Detector}

\subsection{The Bubble Chamber Technique}

The COUPP detectors are continuously-live, mildly superheated bubble chambers, which operate below the threshold for sensitivity to minimum ionizing particles. This simple technology offers an attractive route to low background, low-cost dark matter detectors with multi-ton target masses. Since many fluids can be used in bubble chambers, the spin and mass dependence of the dark matter nuclear coupling could be explored by using a variety of target liquids.

Bubble nucleation in a superheated fluid requires a minimum energy input, which can be supplied by a particle interaction. The additional requirement of achieving a critical energy density in order to nucleate bubbles allows for operating modes which discriminate between different particle interactions on the basis of their specific stopping power (dE/dX). Since nuclear recoils deposit their energy in very short length scales (nanometers) as compared to electrons 
(micrometers) of the same energy, an appropriate choice of operating parameters will result in a bubble chamber that is sensitive to nuclear recoils but blind to minimum ionizing particles and $\gamma$ and $\beta$ interactions. The collaboration has demonstrated that the intrinsic rejection against $\gamma$ interactions is larger than $10^{10}$, at a nuclear recoil threshold of $10 \mathrm{keV}$. This is the same background discrimination mechanism has been used successfully in superheated droplet detectors, such as PICASSO and SIMPLE [4,5].

Although bubble chambers can be made intrinsically insensitive to $\gamma$ and $\beta$ interactions while retaining a high efficiency for nuclear recoils, this is not the case with $\alpha$ interactions. Each $\alpha$ decay has an associated recoiling daughter nucleus, with recoil energy $\sim 100 \mathrm{keV}$ for $\alpha$ decays in the Uranium and Thorium chain. On an event-by-event basis, these decays produce bubbles which are indistinguishable from those produced by WIMP recoils. Two events, correlated in time, are indicative of an $\alpha$ decay chain and can be removed. It is possible to distinguish between a population of multiple $\alpha$ decay events and a population of WIMP events by using energy threshold scanning strategies, but the sensitivity of a WIMP detection experiment is reduced by the need to statistically subtract the background. Therefore, it is important to reduce contamination of the bubble chamber fluids by $\alpha$ emitting isotopes to very low levels. If $\alpha$ contamination can be reduced to the levels that have been achieved in solar neutrino experiments, such as SNO and Borexino [6], it will be possible to improve on our current spindependent dark matter sensitivity by four orders of magnitude.

\subsection{The COUPP-60 Detector}

One virtue of bubble chamber technology is its mechanical simplicity. The detector consists only of a quartz bell jar, a surrounding stainless steel pressure vessel, a bellows arrangement to equalize pressures, a few seals, and fluids. All of these materials are easy to purify or clean, which is crucial given the radiopurity needed to reach relevant dark matter sensitivity levels.

We show a drawing of the chamber in Figure 1 and photos in Figure 2. The superheated liquid $\left(\mathrm{CF}_{3} \mathrm{I}\right)$ is contained in a quartz vessel, with a volume of pure water floating on top. The water isolates the superheated liquid from contact with a set of metal pressure-transmitting bellows. The bellows equalize the pressure inside the quartz with the pressure of the surrounding buffer fluid, thus maintaining low stress in the quartz. The buffer fluid and quartz inner vessel are inside a conventional stainless steel pressure vessel. Pressure control of the superheated liquid is provided by a piston and pump module connected to the buffer fluid. This design allows the pressure to be controlled in a completely hermetic, high purity environment.

The initial target fluid of choice is trifluoroiodomethane $\left(\mathrm{CF}_{3} \mathrm{I}\right)$, which has a density of $2 \mathrm{~g} / \mathrm{cm}^{3}$. Because of its modest boiling point, it is possible to operate a $\mathrm{CF}_{3} \mathrm{I}$ bubble chamber very near atmospheric pressure and room temperature. $\mathrm{CF}_{3} \mathrm{I}$ provides excellent sensitivity to spinindependent couplings because of the large $\mathrm{A}^{2}$ enhancement for scattering on iodine and excellent sensitivity to spin-dependent couplings by virtue of the fluorine which has $\sim 100 \%$ isotopic abundance of $\operatorname{spin} 1 / 2{ }^{19} \mathrm{~F}$ and has a favorable nuclear form factor. 


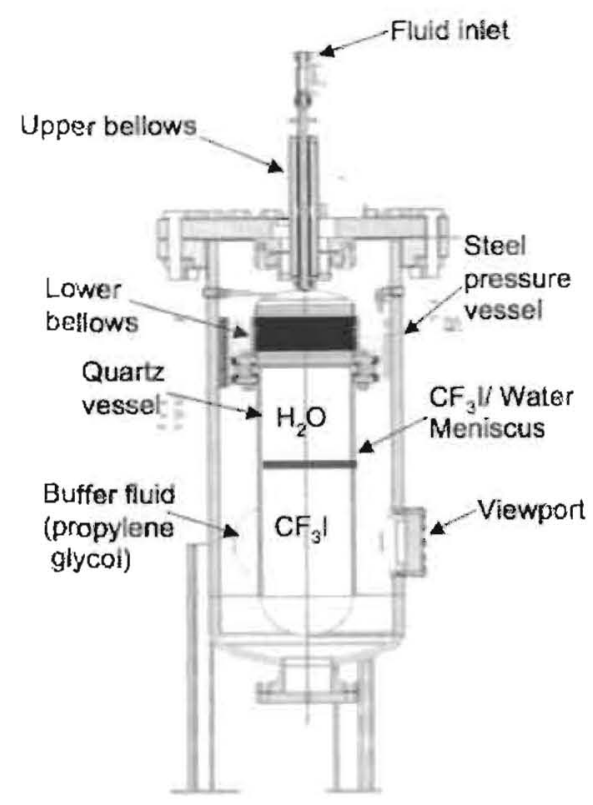

Figure 1: Drawing of the COUPP-60 bubble chamber.
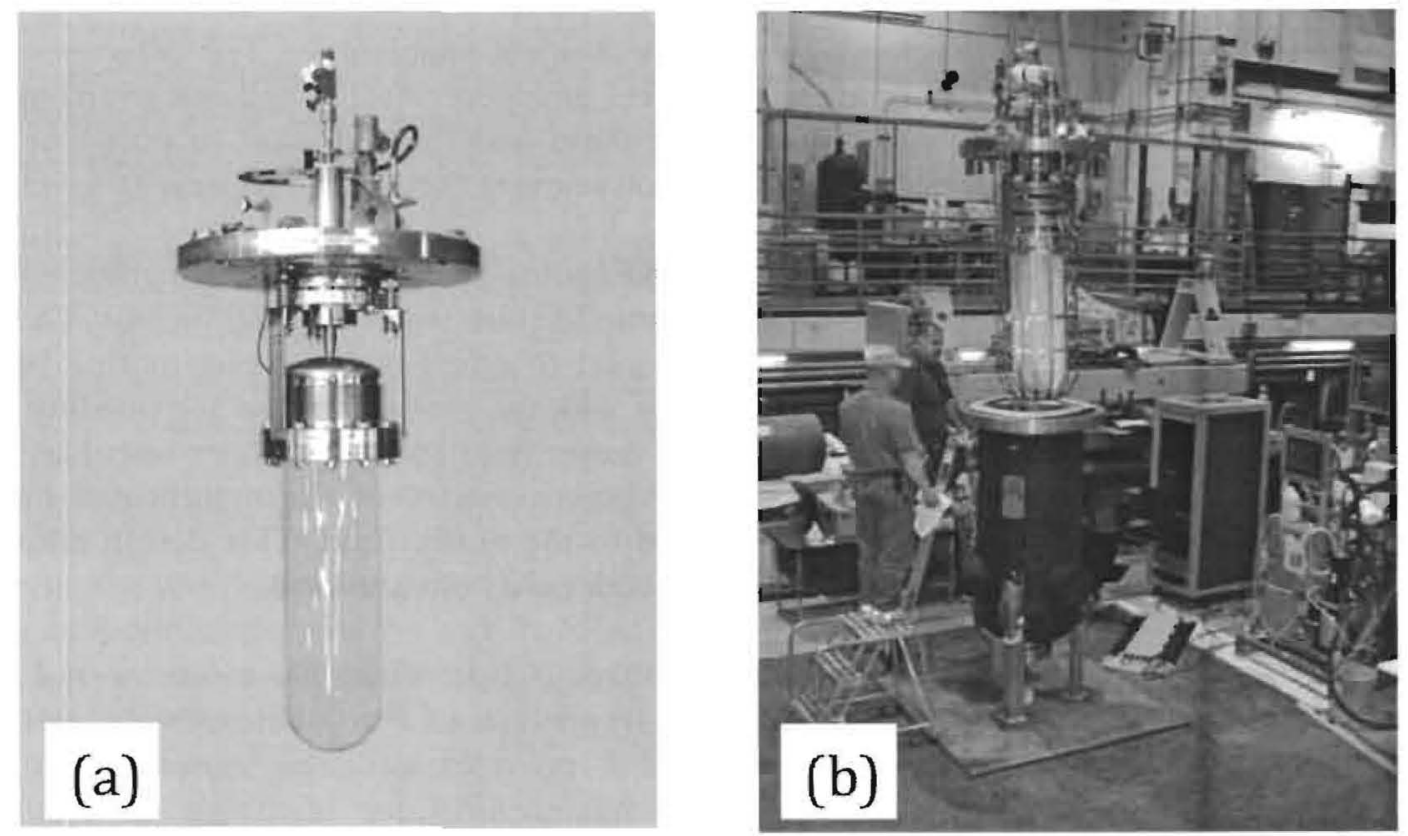

Figure 2: Photos of the COUPP-60 chamber: (a) The inner vessel assembly and (b) the inner vessel assembly being inserted into the pressure vessel. 


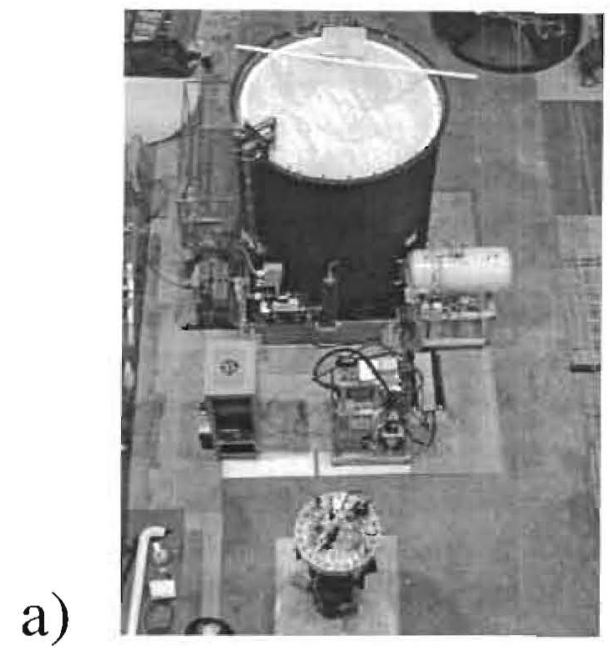

b)

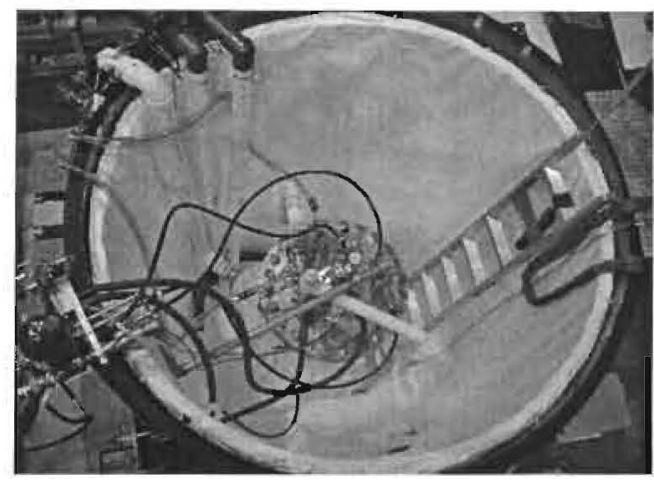

Figure 3. The $60 \mathrm{~kg}$ bubble chamber at the D0 Assembly Hall. a) shows the detector and water shield before insertion. b) shows the detector inside the water tank.

The detector is installed inside an insulated water tank, shown in Figure 3, which provides a shield against environmental neutrons. The temperature of the water, controlled with a heater, is used as the thermal regulator and buffer for the detector. For operation at our shallow commissioning site (the NuMI tunnel at Fermilab), phototubes are floated on a foam raft on the top of the water, turning the tank into a water Cerenkov detector for cosmic ray muon detection.

The sensitive volume inside the quartz inner vessel is viewed by a stereoscopic pair of video cameras. The detector is triggered by comparing each video frame to a reference image. If there are a significant number of pixels that have changed their intensity, then a fast compression of the detector is induced, which forces any bubbles that may have formed to reenter the liquid phase. A set of video frames and chamber status information corresponding to the event is stored. The analysis of these data determines whether a single bubble occurred (WIMPs will never produce multiple scatters) and whether the bubble occurred in the bulk volume, rather than on one of the surfaces of the vessel or at the boundary between the $\mathrm{CF}_{3} \mathrm{I}$ and the water. A fit to the rate of single bubbles as a function of recoil energy threshold separates backgrounds and extracts WIMP cross-section limits.

\section{Technical and Physics Progress, Results, and Goals}

Prior to the COUPP-60 proposal (Fermilab experiment E961) we operated a $2 \mathrm{~kg}$ prototype bubble chamber in NuMI hall as Fermilab test beam experiment T945. This section describes the technical and physics progress made with the 2-kg chamber and shows how those results inform our plans and goals for the next steps. 


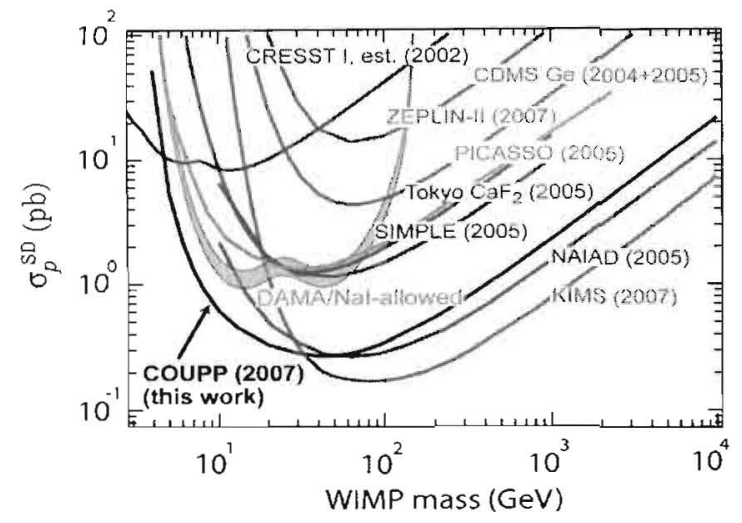

a)

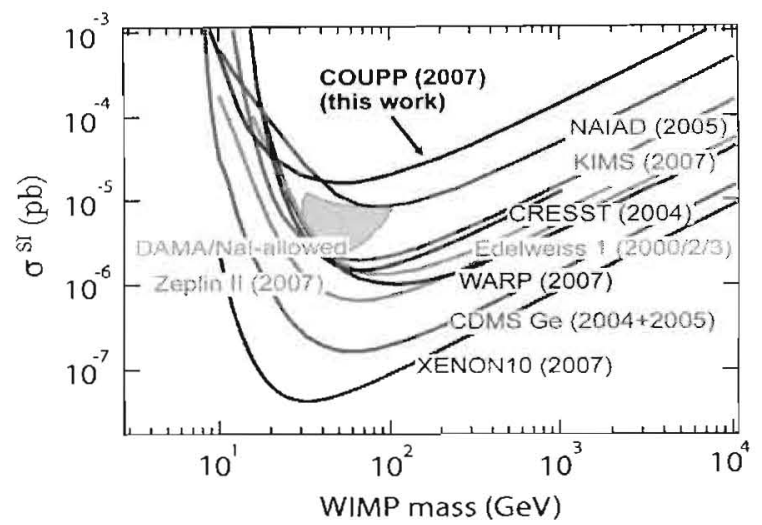

b)

Figure 4. The Coupp dark matter cross-section limits for a) spin-dependent proton (left) and b) spin- independent scattering published in Science, Feb 2008[7].

\subsection{T945 ( $2 \mathrm{~kg}$ chamber) results published in Science}

We reported best-in-the-world upper limits for proton spin-dependent dark matter nucleus scattering in February 2008 [7]. These results, shown in Figure 4a, were limited by backgrounds from radon contamination at the 77 events $/ \mathrm{kg}$-day level. The source of this contamination (an Oring seal) was identified and replaced in an upgrade. Due to this background, our spinindependent results, shown in Figure 4b, were several hundred times above the then best limits from Xenon-10 and CDMS.

\subsection{Subsequent results by other groups since our 2008 publication}

In 2009 CDMS published improved spin independent limits [8] about a factor of 4 lower than shown in Figure 4b. The minimum of their new limit curve matches Xenon-10's minimum shown above, but at a higher mass $(60 \mathrm{GeV})$. The PICASSO experiment has just submitted for publication [9] new spin-dependent limits about a factor of 4 below the COUPP limits shown in Figure 4a. Other groups continue spin independent searches using liquid noble gas detectors and solid state detectors. Progress is being made but no new results have appeared in publication.

\subsection{Subsequent results from the $2 \mathrm{~kg}$ chamber}

We modified the 2-kg chamber by replacing the O-ring seal with a teflon-coated inconel seal measured to have low-levels of Radon emanation. At the same time, the quartz jar was replaced with a new, acid-etched and precision cleaned jar. Ultra-high-purity water from SNOLAB was used to fill the detector. We took data again in NuMI hall with this modified chamber in late 2007 through early 2008. The bulk bubble rate dropped to the 10 events/kg-day level, confirming that the O-ring was indeed a substantial source of radon. The remaining events are from $\alpha$ decays 
and cosmic ray induced neutrons, with the absence of time correlations between events eliminating radon as a major component. This observation of a residual $\alpha$-decay rate led to investments in a substantial improvement in the fluid handling and purification system for the 60 $\mathrm{kg}$ chamber.

The rate of deadtime-causing wall bubbles remained unchanged indicating that the source of the $\alpha$ decays nucleating those bubbles was in the bulk quartz rather related to surface effects. This has led us to replace natural quartz jars with synthetic silica where the contamination from uranium and thorium daughters is known to be 2-4 orders of magnitude lower. Early results from our new prototype chambers are confirming this hypothesis.

We took a much larger data set with our neutron source to improve the calibration of the chamber. These data confirmed our earlier neutron calibration. Simulations of these results, shown in Figure 5, indicated excellent agreement with the observed rate of neutron induced single bubbles and confirm, to the better than 10 percent level, both that our threshold energy scale is accurate and that our efficiency to produce, and detect, a single bubble is $100 \%$. There is also fair, but not precise agreement with the rate of neutron produced multiple bubble events. We believe that the disagreement is mostly due to the inefficiency of our bubble finding code for multiple bubble events.

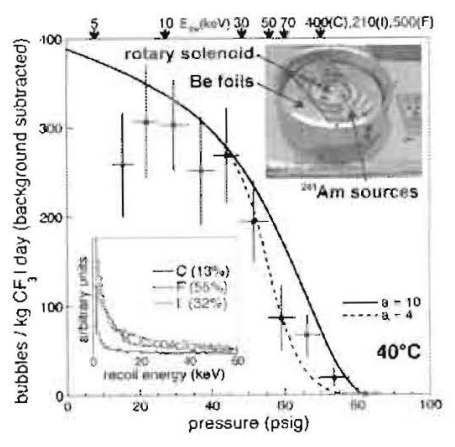

a)

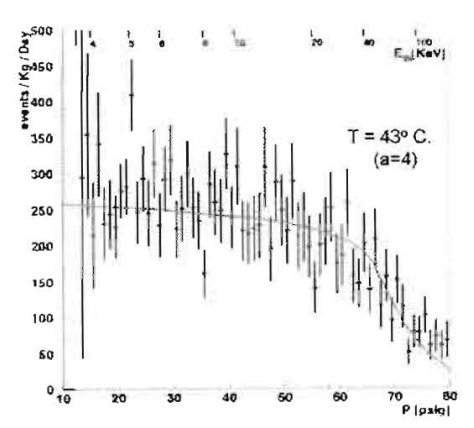

b)

Figure 5. AmBe neutron induced single bubble rate and apriori simulation: a) shows early work, while b) shows high statistics 2007 data. Translation from pressure to threshold energy is shown on the upper scales for the two temperature settings.

We studied the response of the chamber to electromagnetic background using a sequence of $\gamma$ ray sources. Our detector is very advantageous in that the bubble nucleation mechanism is insensitive events, arising from $\gamma$ and $\beta$ interactions in the fluid, to a very high level because of the far lower ionization density as compared to nuclear recoils. The rejection of bubbles induced by electromagnetic backgrounds exceeds $10^{10}$ at a threshold of $10 \mathrm{KeV}$. The extrapolated electromagnetic background rate at this threshold, for the conditions found in the NUMI tunnel, is $\sim 0.01$ events $/ \mathrm{kg}$-day. We expect the pure water shield at SNOLAB to reduce this level even further. 
We built a muon veto around the detector to tag bubbles where the neutron was produced by a cosmic ray muon interacting inside the detector. The rate of the veto $(>100 \mathrm{~Hz})$ and the inherent uncertainty in the visual timing of the bubble formation prohibited us from using this device alone as a veto. By relying on acoustic sensors to provide a fast bubble timing signal we could measure the cosmic muon induced signal in the low threshold region where bubbles were loud enough to be heard. About half ( $5-10$ events/kg-day) of the single bubbles at low threshold are induced by cosmics. Unlike $\alpha$ induced bubbles, cosmic induced neutron interactions can produce an integral energy spectrum resembling that from heavy WIMPs. For that reason, it is especially critical to minimize the number of these events.

\subsection{COUPP-60 goals in Fermilab's NuMI Hall}

The primary goal for operating the $60 \mathrm{~kg}$ chamber in NuMI hall is to demonstrate full, stable, remote operations. The muon system built for COUPP-60 is designed to be very efficient and have negligible rates due to environmental radiation other than muons, with an accidental counting rate expected to be $\sim 1 \mathrm{~Hz}$. Cosmic rays crossing within the water shield are expected to be $\sim 10 \mathrm{~Hz}$. With this system we will be able to characterize, with a large sample, the detailed properties of cosmic ray induced events from those tagged by the muon system. We plan to simulate this background to the best of our ability. We estimate the untagged cosmic induced event rate to be below 0.1 events/kg-day.

At NUMI we plan to achieve less than 1 event $/ \mathrm{kg}$-day of single bubble events from $\alpha$ decays in the bulk and any other sources. Depending on how well the fluid handling and purification system performs this rate could be substantially lower. Reaching even this modest goal will result in significantly higher sensitivity than our published result.

\subsection{COUPP-60 at Depth}

Once the goals above are achieved we will want to move deep underground. With more rock overburden the signal from cosmic rays will be negligible for a run of any reasonable duration with a $60 \mathrm{~kg}$ chamber. As discussed in detail in Section 4, a background problem we know is ahead are bulk bubbles from $\alpha$ decaying Radon daughters left in the working fluid. For example, in the past we have introduced $\sim 1000 \mathrm{Radon}$ atoms $/ \mathrm{kg}$ into the chamber when filling. If we achieve 100 radon atoms $/ \mathrm{kg}$ when filling the $60 \mathrm{~kg}$ chamber then the rate of single bulk bubbles from the Po-210 in that decay chain will produce 0.01 events $/ \mathrm{kg}$-day.

The state of the art in purification of $\alpha$ emitters from bulk materials is at the 0.01 events $/ \mathrm{kg}$-day level [10]. Our 2007 data was about 3 orders of magnitude worse than that. We have considerable headroom before we must improve the state of the art. As an example of how we can improve our background rejection, Section 4.4 discusses results from PICASSO that show that $\alpha$ decay events in a superheated liquid can be identified by their acoustic signature.

A background at the 0.01 events $/ \mathrm{kg}$-day level would yield only of order 100 events in a 6 month run. This is low enough for excellent dark matter limits but marginal in terms of characterization of a background source. When we have controlled backgrounds to this level it will be time for either a bigger or more chambers. 


\subsection{COUPP-60 Goals Summary}

In Table 1 we summarize the sensitivity goals described above. In Figure 6 we show the spin dependent and spin independent dark matter cross-section limits that correspond to an observed count rate of 0.01 events/kg-day, assuming all observed events are signal candidates. We measure and fit an integral recoil energy spectrum and thus will always be able to set limits below that shown. The actual improvement factor depends on the spectrum observed, the nature of the background that caused those events, and our exposure. In our 2008 publication we were able to confidently identify the background as $\alpha$ decays from the Radon decay chain. This allowed us to make a background subtraction in the fitting that increased the sensitivity (and lower our limits) by a factor of 10 .

\begin{tabular}{|c|l|}
\hline $\begin{array}{c}\text { Sensitivity Limit } \\
\text { Untagged Bulk bubbles }\end{array}$ & \multicolumn{1}{|c|}{ Milestone } \\
\hline $77 / \mathrm{kg}$-day & $\begin{array}{l}\text { The background rate from Radon measured in work published } \\
\text { in Science magazine. }\end{array}$ \\
\hline$\sim 10 / \mathrm{kg}$-day & The rate achieved in 2007 run after Radon leak mitigation. \\
\hline$\sim 1 / \mathrm{kg}$-day & $\begin{array}{l}\text { Goal of COUPP-60 at NuMI. At this time we are ready to go } \\
\text { deep underground. }\end{array}$ \\
\hline $0.1 / \mathrm{kg}$-day & $\begin{array}{l}\text { Untagged cosmic neutron induced backgrounds become } \\
\text { important at NuMI. }\end{array}$ \\
\hline$\sim 0.01 / \mathrm{kg}$-day & $\begin{array}{l}\text { Potential reach for alpha contamination using the improved } \\
\text { fluid handling system. } \gamma \text { 's become a potential background issue } \\
\text { at the lowest thresholds. }\end{array}$ \\
\hline$<0.01 / \mathrm{kg}$-day & $\begin{array}{l}60 \text { kg detector is too small to statistically characterize } \\
\text { backgrounds }(<0.5 \text { event /day } ;<100 \text { events/run). }\end{array}$ \\
\hline$<0.001 / \mathrm{kg}$-day & $\begin{array}{l}\text { Neutron induced bubbles from either rock or cosmic neutrons at } \\
\text { depth. }\end{array}$ \\
\hline
\end{tabular}

Table 1. List of sensitivity benchmarks for COUPP 

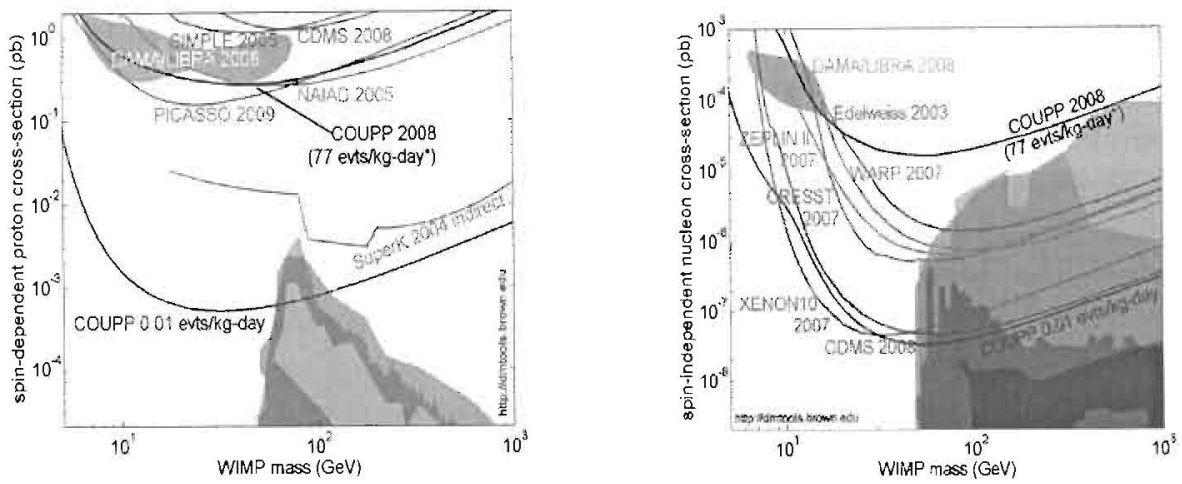

a)

b)

Figure 6. Projected limits for a) spin-dependent proton (SDP) and b) spin independent dark matter cross-sections, assuming a background rate of 0.01 events/kg-day and that all background events are considered signal. Fitting the integral spectrum will lower the limits shown.

\section{Background Events in the COUPP Bubble Chamber}

As with all dark matter experiments, the major difficulty in COUPP is in reducing, controlling and understanding background events, unassociated with nuclear recoils arising from dark matter interactions. Our inherent electron rejection means that the dominant backgrounds in our experiment will come from $\alpha$ decays in the active volume (primarily from Radon and daughter atoms) and neutrons coming from the environment. To reduce Radon contamination we pay close attention to keeping the active volume radio-pure, including aggressive cleaning procedures. This imposes strict conditions on how the detector is assembled. Neutron interactions in our target come from two sources: $(\alpha, n)$ processes from radioactive decay in the surrounding material, and cosmic ray induced neutron production. Shielding of the detector with pure materials reduces the former, while installation deep underground is necessary to reduce the latter. Low energy neutrons do not give high enough recoil energy to the target nuclei to induce bubble formation.

\subsection{Radiopurity of the inner vessel}

We have found a rate of 250 events/day coming from the walls of the inner quartz vessel in the 2 $\mathrm{kg}$ detector. Most of this rate is from the naturally occurring Uranium and Thorium content of the natural quartz we used. Paying particular attention to cleaning the vessel and keeping it isolated from the atmosphere did not reduce this rate. We are addressing this problem in COUPP-60 by using synthetic quartz for the inner vessel. From measurements of Uranium content in quartz and from initial tests on a smaller synthetic quartz chamber, we expect at least 
two orders of magnitude reduction in the wall rate. Since it is easy to tag an event as arising from the wall, this background will not affect our sensitivity. Wall events do contribute to the dead time of the detector but, at the levels we expect with the new synthetic quartz vessels, this will not affect our exposure significantly

We will be assembling the cleaned inner vessel in a Class 100 clean room at Fermilab. With this capability and attention to aggressive cleaning of the fluid handling system, we plan to reduce the non-cosmic bulk bubble event rate by at least an order of magnitude, to $<1$ events/kg-day at NUMI and less at SNOLAB.

\subsection{Environmental Neutron Background from Radioactivity}

The background from environmental radioactivity for an unshielded detector has a rate of $\sim 15$ events $/ \mathrm{kg}$-day. We have approximately 1 meter of water and propylene glycol surrounding the inner vessel. This thickness of fluid will provide a reduction of about $10^{5}$ against environmental neutrons, reducing the rate of this background to irrelevancy.

\subsection{Cosmic Ray Associated Neutron Background.}

Cosmic rays have a soft hadronic component at the surface that can induce bubble nucleation in the detector. This background is eliminated even at the shallow depth of the NuMI laboratory. Of more difficulty are the higher energy neutrons generated by high energy muon interactions in the material surrounding the bubble chamber. This background scales roughly linearly with the cosmic ray flux, although the spectrum of generated neutrons becomes harder at greater depth. One of the difficulties in handling this background is that the energy spectrum of the generated neutrons gives a shape to the spectrum, for which a realistic model has to be developed for the fit. If this background can be eliminated through sheer depth, then no background subtracting fit is necessary.

\subsection{Acoustic Discrimination of $\alpha$ Decay Events}

Recently the PICASSO collaboration has reported evidence for the discrimination of $\alpha$ and neutron induced bubbles by their acoustic signature [11]. This is possible in principle, because $\alpha$ particles make tracks $\sim 100$ times longer than nuclear recoil tracks, nucleating multiple small bubbles, which grow together on longer time scales. Simply put, $\alpha$ 's are louder than nuclear recoils. We are implementing the COUPP detector with several acoustic sensors in the hopes that this technique can be applied on our chambers. Depending upon the efficiency of this separation, this will allow us to either relax the purification requirements or achieve a higher sensitivity at a given $\alpha$ contamination level.

\subsection{Neutron backgrounds in the NuMI tunnel.}

The muon system built for COUPP-60 is designed to be $99 \%$ efficient with an accidental counting rate of $\sim 1 \mathrm{~Hz}$. With that system at NuMI, we will be able to characterize, with a large sample, the detailed properties of cosmic ray induced events. This is not possible at the surface where the deadtime from cosmic-ray-induced events would be prohibitive. 
We have found a cosmic-ray-induced background rate of 5-10 events/kg-day in the NuMI near detector tunnel, using the data from the $2 \mathrm{~kg}$ chamber. This rate is an order of magnitude larger than the bulk background rate we hope to achieve at that site. The muon veto we have built will tag a large fraction of these cosmic induced events. But even a $1 \%$ inefficiency in vetoing will result in a significant $(\sim 0.1$ events $/ \mathrm{kg}$-day) background rate at NuMI. In a 6 month run at NuMI we might see $\sim 1000$ unvetoed cosmic ray neutrons. When our non-cosmic backgrounds are below 1 bulk events $/ \mathrm{kg}$-day, the uncertainty in the untagged cosmic induced rate will become significant and this fact will drive us to move to a deep underground site.

\subsection{Neutron backgrounds at SNOLAB.}

The reduction in cosmic ray rate at SNOLAB, at a depth of $2 \mathrm{~km}$, is approximately $4 \times 10^{6}$. The cosmic ray background can be ignored. No muon veto would be required since a 6 month run would likely have no cosmic events. The sensitivity improvement achievable there will likely be limited by other backgrounds. When these are characterized with data we can make concrete plans to reduce them.

\section{The COUPP $60 \mathrm{Kg}$ Installation at SNOLAB}

\subsection{Site Requirements}

The COUPP underground installation must meet the following requirements.

\subsubsection{Water shield.}

A water shielding tank is required with inner diameter $\sim 7^{*}$ and height $\sim 9^{\prime}$. The water must be temperature controlled to be 0.1 degree $\mathrm{C}$ stability, with the temperature adjustable between 25 degrees $\mathrm{C}$ and 45 degrees $\mathrm{C}$.

\subsubsection{Water}

Filling the tank will require approximately 4000 gallons of filtered water. We envision several fills being needed during a year's operation. The water must be chlorinated or otherwise treated to avoid bacterial growth. We need a place to drain this amount of water so that we can access the detector.

\subsubsection{Electrical power}

We require:

Two $120 \mathrm{~V}, 20 \mathrm{~A}$ circuits for the water pump and heater

One 120V, 20A circuit for the chiller

Two $120 \mathrm{~V}, 15 \mathrm{~A}$ circuits for powering miscellaneous equipment

\subsubsection{Compressed Air}

A single compressed air line is needed, with 100 psi pressure and 4 SCFM flow. We also require a compressed nitrogen gas cylinder. 


\subsubsection{Clean environmental conditions}

At least class 10,000 clean conditions need to surround the detector to allow small modifications to the piping and sample extraction without contaminating high-purity fluids.

\subsubsection{Footprint, height and floor loading}

A flat area of $24^{\prime} \times 14^{\prime}$ is required. The total weight that needs to be supported, including the full water tank, is 20 tons. The minimum height required is about $20^{\prime}$, depending on the design of the crane (see below).

\subsubsection{Networking}

A single computing network link of at least $100 \mathrm{MBit} / \mathrm{s}$ transfer speed is required.

\subsubsection{Crane}

An overhead crane or gantry is needed for moving the detector into and out of the water tank. The minimum height required to the crane hook is equal to the height of the water tank (124") plus the length of the inner or outer assembly ( $88^{\prime \prime)}$, or about 18 feet. The capacity should be 3 tons.

\subsubsection{Safety}

An analysis of the hazards of the experiment at its final location must be made and any necessary mitigation will have to be addressed. For the SNOLAB site, recertification of our pressure components will need to be made in accordance with applicable Canadian codes.

\subsection{Ladder Labs}

After a preliminary analysis of our site requirements, SNOLAB staff have recommended the $F$ site of the Ladder Labs as a suitable location for the installation. Figure 7 shows a sketch of the water tank situated at this location, with a hoist lifting the inner vessel out of the tank. The ceiling height at this location is 23 feet. Note that the inner vessel assembly of the bubble chamber and the pressure vessel must be removed separately from the water tank in this location, due to height constraints. This requires a minor deviation from the assembly procedure used at the Fermilab commissioning sites, which have more overhead clearance.

The following upgrades would be required to the Ladder Labs site:

- A new water shield tank must be constructed, since the water tank used for commissioning at Fermilab can not be moved underground.

- Water for filling the shield tank would be taken from SNOLAB high purity water plants. A new connection would have to be made to a water line that is already planned to pass near the Ladder Labs.

- Electrical circuits need to be laid into the Ladder Labs from a nearby distribution box.

- Lights would have to be installed.

- A compressed air line would need to be installed. A line runs past the Ladder Labs.

- A network cable would need to be installed.

- A monorail crane would need to be installed. 
- A fire alarm system would need to be installed.

The costs of the new water tank and needed improvements to the ladder labs have been analyzed and estimated to be $253 \mathrm{k} \$$ for equipment and $207 \mathrm{k} \$$ for labor, including a $50 \%$ contingency factor.

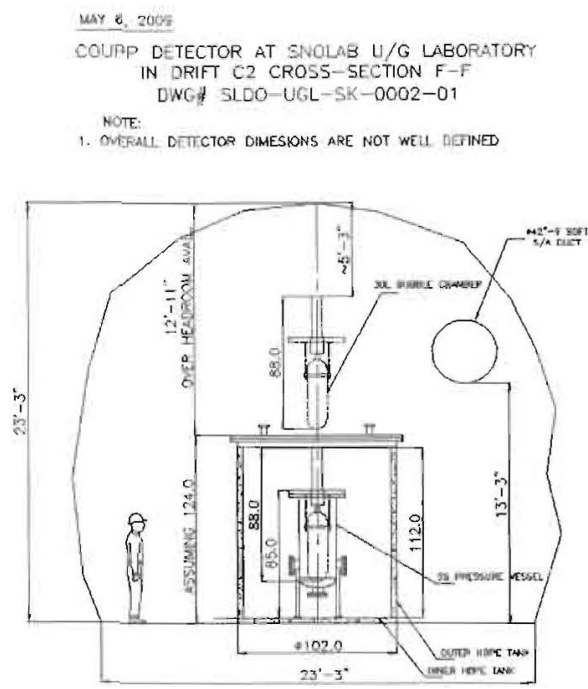

Figure 7. The water shielding tank and bubble chamber at location $\mathrm{F}$ of the Ladder Labs.

\section{Schedule}

The COUPP-60 experiment is currently at a quite advanced stage of construction. All components are set up in the D0 assembly pit at Fermilab, as shown in Figure 3, and laboratory safety committees have approved the operation of the detector. This run at D0 is a system integration test, to insure that the separately tested pieces of the system function well together. By Fall, 2009, the experiment should be ready to be deployed underground in the NuMI tunnel. At that time we will replace the inner vessel used for engineering runs with a precision cleaned, high-purity version with a synthetic quartz vessel. The move itself should take on the order of one month. The duration of tests at NuMI depends considerably on the level of background we observe. If we achieve a bulk background level of 1 event/kg-day, then we will quickly become dominated by uncertainties in the cosmic induced rate and we will want to move as soon as possible to a deep site. Alternatively, improvements to the inner vessel and fluid handling system would be indicated if the $\alpha$ backgrounds are higher than expected. 
A time line for the experiment is:

$\begin{array}{ll}\text { TIME LINE } \\ \text { FY09 } & \begin{array}{l}\text { Complete fabrication and testing at D0 } \\ \text { Commissioning and operation at NuMI, } \\ \text { Installation of underground infrastructure at } \\ \text { SNOLAB }\end{array} \\ \text { FY11 } & \begin{array}{l}\text { Recommission experiment deep underground, } \\ \text { begin running } \\ \text { Operations deep underground }\end{array}\end{array}$

\section{Required Proposals and Approvals}

This proposal is one of three required for us to move ahead with our plan to operate the COUPP60 Bubble chamber at SNOLAB. In addition to the approval of SNOLAB we must propose and be approved and funded by both Fermilab and the US Department of Energy. Fermilab charged us to evaluate deep underground sites for suitability. Both the SOUDAN mine and SNOLAB were judged acceptable. Our collaboration strongly prefers SNOLAB because the depth is sufficient to eliminate cosmic ray induced events as a significant background, there are resources and expertise at SNOLAB to help with mitigation of $\alpha$ emitter contamination, and there is the potential at SNOLAB for collaboration with other experts in the field. This judgment has led us to submit this proposal. We are preparing the Fermilab and DOE proposals now, and will provide those documents as they become available. A recently approved DUSEL S4 proposal describes the deployment of the $60 \mathrm{~kg}$ chamber at SNOLAB and includes support for this activity. 


\section{References}

[1] Roszkowski, et.al.; JHEP 0707:075 (2007)

[2] Bertone, et.al,; Phys. Rev. Letters 99, 151301 (2007)

[3] COUPP website http://www-coupp. fnal.gov/

[4] Phys.Lett.B624:186-194,2005

[5] Phys.Lett.B621:233-238,2005

[6] Nucl.Instrum.Meth. A517 (2004) 139-153; Phys.Rev.Lett.101:091302,2008

[7] Science 319, 933(2008).

[8] Ahmed, et.al., Phys.Rev.Lett 102, 011301 (2009).

[9] Archambault, et.al., arXiv:0907.0307v1 [hep-ex] 2 Jul 2009.

[10] F.Aubin, et.a1., Discrimination of nuclear recoils from $\alpha$ particles with superheated liquids, (PICASSO) 10 (2008) 103017, 16 October 2008, New Journal of Physics

[11] C. Arpsella et al., Phys. Lett. B 658, 2008. 


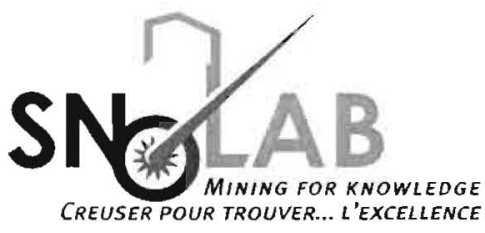

Dr. Juan Collar

COUPP Spokesman

University of Chicago,
Office for Operations

Queen's University

99 University Ave

Kingston, ON Canada K7L 3N6

Tel: (613) 533-2679

Fax: (613) 533-6813

21 July, 2009

Re: Installation of COUPP at SNOLAB

Dear Dr's Collar and Sonnenschein;

Thank you for your continued interest in siting the COUPP experiment at SNOLAB, and for providing more detail on the infrastructure requirements and schedule for its potential installation.

Should you select SNOLAB as the site for COUPP, SNOLAB would be very pleased to work with you and your collaboration with a goal of getting COUPP installed in the SNOLAB facility in the coming year. We have already made significant progress in evaluating the infrastructure needs of the project, and will be prepared to assist COUPP in the following ways:

- You have identified a number of specific infrastructure items that would be required for a successful implementation, including a water shielding tank, a 3-tonne gantry crane, and a variety of services including ventilation, compressed air, utility water, ultra pure water, drains, electrical power, networking and communications. Our estimate for infrastructure amounts to $\sim \$ 460,000$, including labour, on site transportation costs and contingency. This assumes we supply the labour using in house technical staff. The details of these costs have been carefully processed and are tabulated in the excel spreadsheets developed by you and Dr Fraser Duncan. For reference, an overview of the breakdown is as follows:

- A water shielding tank complete with liner, custom designed lid, insulation, and ladders for access. The estimated materials cost with transportation is $\$ 86,000$.

- Gantry crane and associated components: $\$ 31,000$

- Basic Hall infrastructure, material costs (including ventilation, compressed air, utility water, ultra pure water, drains, electrical power, networking, communications, and miscellaneous): $\$ 41,000$

- Miscellaneous tools and furniture to establish a rudimentary control room and have basic supplies: $\$ 13,000$

- Integration of a fire alarm system appropriate for this installation: $\$ 82,000$.

- The labour cost for engineering design, and physical installation is estimated to be $\$ 207,000$. 
The total cost of the experiment related infrastructure listed above is estimated to be $\$ 171,000$. On behalf of SNOLAB I am prepared to commit to support these items at that level, assuming the COUPP collaboration makes a firm commitment to site the detector in SNOLAB. The total budget for these items will be capped at $\$ 200,000$. In addition, we will provide the fire alarm system (estimated cost $\$ 82,000$ ), and make an in-kind contribution by supplying resources to cover the labour associated with the installation. This is an estimated in-kind contribution of $\$ 207,000$.

- We will provide engineering resources for the design and installation at SNOLAB.

- We will provide technical support for the installation. We have carefully considered the manpower required, and we feel this can be done with the resources already on staff at SNOLAB. If the installation requires more resources than we have available, the costs of the additional manpower would have to be supplied by COUPP.

- SNOLAB has an active Research Scientist program and generally we are able to arrange for at least one Research Scientist to participate with each experiment as a local contact and facilitator. In many cases the research scientists will become full collaboration members. This will need to be negotiated with the Research Scientists.

SNOLAB feels privileged to be able to offer this level of support: scientific and technical support as an institutional collaborator, and financial support $(\$ 460,000$ in case of COUPP) to help establish the COUPP scientific program at SNOLAB. We hope that you are able to come to a positive decision soon, in part as the equipment support is tied to a budget that requires all expenditures be completed this calendar year. We look forward to working with you to develop a plan finalizing the resources required, the physical layout, and other engineering details. This will enable us to expedite the necessary purchases while the funds are available.

As you know, there will be a SNOLAB workshop and Experimental Advisory Committee (EAC) Meeting in Sudbury, August $27^{\text {th }}$ and $28^{\text {th }}, 2009$. We will look forward to a presentation of the COUPP proposal to the EAC at that time. The installation in SNOLAB will be contingent on receiving a favourable review by the $\mathrm{EAC}$. In addition, the experiment will need to undergo a technical and safety review organized by SNOLAB.

SNOLAB is very excited about the prospects for COUPP at SNOLAB and looks forward to working with you to help make it a reality.

Yours sincerely

Tony Noble

Director SNOLAB

Cc: Nigel Smith (SNOLAB Director-Elect), Fraser Duncan (Deputy Director SNOLAB)

David Sinclair (SNOLAB Facility Development Director)

Member Institutions

University of British Columbia, Carleton University, University of Guelph, Laurentian University, University of Montreal, Queen's University 